Bundesgesundheitsbl 2013 $\cdot 56: 474-475$

DOI 10.1007/s00103-012-1647-4

๑ Springer-Verlag Berlin Heidelberg 2013

\title{
Mindestanforderungen an die mikrobiologische Kontrolle von Blutkomponenten zur Transfusion - Aktualisierung des Votums 16
}

\section{Bei der 74. Sitzung des Arbeitskreises Blut am 07.11.2012 wurde folgendes Votum (V 43) verabschiedet:}

Mit der vorliegenden Überarbeitung des Votums 16 des Arbeitskreises Blut „Mindestanforderungen zur Sterilitätstestung von Blutkomponenten" (V 16) soll den seit 1997 gewonnenen Erkenntnissen aus der mikrobiologischen Kontrolle von Blutkomponenten Rechnung getragen werden. Da diese Arzneimittel nicht sterilisierbar sind, eine Endproduktspezifikation ,steril“" mithin nicht zu fordern ist, wird der Begriff „mikrobiologische Kontrolle" anstelle "Sterilitätstestung“ verwendet. Eröffnet wurde die Möglichkeit, als Bezugsgröße N zur Berechnung der zu testenden Stichprobe die Anzahl der pro Monat in einer Betriebsstätte durchgeführten Verfahren anstelle der hergestellten Einheiten eines Präparatetyps zugrunde zu legen Grundsätzlich kann eine relevante prädiktive Information zur mikrobiologischen Qualität der Grundgesamtheit der hergestellten Einheiten nicht erhalten werden, wenn lediglich $1 \%$ oder $0,4 \times \sqrt{ } \mathrm{N}$ der pro Monat hergestellten Einheiten getestet werden. Es ist daher angemessen, der Bezugsgröße N die Anzahl der Verfahren und nicht der hergestellten Präparate zugrunde zu legen.

Weitere Änderungen betreffen die verbindliche Durchführung einer zweiten Kultur bei positivem Signal sowie den Erfahrungen angepasste Vorgaben für die Entnahme und Aufbewahrung des Untersuchungsmaterials.

\section{Gewinnung des Untersu- chungsmaterials - Zeitraum}

Untersuchungsmaterial sind bei zerstörender Prüfung die Blutkomponenten selbst.

Falls das Präparat noch transfundiert werden soll, ist die Entnahme von Aliquots des Untersuchungsmaterials im funktionell geschlossenen System (durch steriles Anschweißen eines Probenbeutels mit Dichtigkeitstest bzw. über einen bereits im Set integrierten Probenbeutel) erlaubt. Das Volumen des Aliquots muss eine Bestätigungstestung erlauben; die Spezifikationen bezüglich Volumen und Gewicht müssen erfüllt bleiben. Die Zeiträume für die Gewinnung des Untersuchungsmaterials sind so gewählt, dass mit hoher Wahrscheinlichkeit eine Kontamination nachgewiesen werden kann (• Tab. 1).

\section{Gewinnung des Untersu- chungsmaterials - Häufigkeit}

Die Anzahl N für die Qualitätskontrolle an Stichproben bezieht sich immer

\begin{tabular}{|ll|}
\hline Tab. 1 & $\begin{array}{l}\text { Zeitraum für die Gewinnung des Untersuchungs- } \\
\text { materials }\end{array}$ \\
\hline Erythrozytenkonzentrat (EK) & $\begin{array}{l}\text { Frühestens 10 Tage nach Entnahme, spätestens 3 Tage } \\
\text { nach Ende der Haltbarkeit }\end{array}$ \\
\hline Thrombozytenkonzentrat (TK) & $\begin{array}{l}\text { Frühestens 24 } \mathrm{h} \text { vor Ablauf der Haltbarkeit, spätestens } \\
72 \mathrm{~h} \text { nach Ablauf der Haltbarkeit }\end{array}$ \\
\hline Plasma aus Apherese für Therapiezwecke & $\begin{array}{l}\text { Nach Apherese und anschließender Lagerung für } \\
\text { mindestens 96 } \mathrm{h} \text { bei Raumtemperatur }\end{array}$ \\
\hline Autologes Vollblut, autologes EK & $\begin{array}{l}\text { Spätestens 10 Tage nach Ende der Haltbarkeit aus nicht } \\
\text { transfundierten Einheiten }\end{array}$ \\
\hline
\end{tabular}

auf die monatlich in einer Betriebsstätte durchgeführten Verfahren. Die Verluste an nicht häufig hergestellten Blutkomponenten sollten möglichst gering gehalten werden. Bei weniger als 400 Verfahren pro Monat sind mindestens 4 Präparate pro Monat zu testen, bis zu 1500 Verfahren einer Art pro Monat sind 1\% von $\mathrm{N}$ zu testen. Betriebsstätten, die mehr als 1500 Verfahren einer Art pro Monat durchführen, dürfen die Häufigkeit der Probenentnahme mit $0,4 \times \sqrt{ } \mathrm{N}$ festlegen. Die für die jeweiligen Verfahren zu testenden Komponenten sind in $\bullet$ Tabelle 2 zusammengefasst.

Für Plasma aus der Vollblutentnahme kann die mikrobiologische Kontrolle entfallen, da mögliche Kontaminationen bei der Vollblutentnahme bereits durch die mikrobiologische Kontrolle der EK bzw. Pool-TK erfasst werden und Plasma keine weiteren kritischen Herstellungsschritte erfährt.

Für EK, TK und Plasma aus der Aphereseverfahren.

Für Blutkomponenten aus der Multikomponenten-Apherese ist $\mathrm{N}$ ebenfalls rese ist $\mathrm{N}$ die Anzahl der jeweiligen Aphe- 


\section{Tab. 2}

\begin{tabular}{|ll}
\hline Verfahren, auf das sich die Bezugsgröße $\mathbf{N}$ bezieht & Zu testende Komponenten \\
\hline Herstellung aus der Vollblutspende & EK \\
\hline Herstellung von Pool-TK & Pool-TK \\
\hline Erythrozytapherese & EK \\
\hline Thrombozytapherese & TK \\
\hline Plasmapherese & Plasma für Therapiezwecke \\
\hline Multikomponentenspenden aus Apherese mit TK-Herstellung & TK \\
\hline Multikomponentenspenden aus Apherese ohne TK-Herstellung & EK \\
\hline Verfahren zur präoperativen Eigenblutgewinnung & EK bzw. Vollblut \\
\hline
\end{tabular}

die Anzahl der Verfahren, wobei die mikrobiologische Kontrolle, wie oben für die Vollblutentnahme beschrieben, an der jeweils sensitivsten Komponente (TK oder EK) durchzuführen ist, die mit diesem Verfahren hergestellt wird.

Bei Rezeptur-Herstellung aus Blutkomponenten (z. B. gewaschene EK, rekonstituiertes Vollblut) trifft der Hersteller die Entscheidung über zusätzliche mikrobiologische Kontrollen in Abhängigkeit von der Komplexität des Herstellungsverfahrens entsprechend seiner Risikoanalyse.

Bestrahlte Blutkomponenten sowie Blutkomponenten, die im funktionell geschlossenen System geteilt wurden, erfordern keine mikrobiologischen Stichprobenkontrollen zusätzlich zu denen der Ausgangsverfahren.

\section{Präanalytik für die mikrobiologische Kontrolle}

Das Untersuchungsmaterial kann vor Beimpfung der Kulturflaschen bis zu 3 Tage bei $4-28^{\circ} \mathrm{C}$ aufbewahrt werden. Die Probenentnahme für die Kultur erfolgt aseptisch nach sorgfältiger Durchmischung des Untersuchungsmaterials (Blutkomponente oder Aliquot). Es ist darauf zu achten, dass sowohl für die Desinfektion der Probenentnahmestelle als auch des Septums der Kulturflasche ein geeignetes Desinfektionsmittel z. B. aus der Liste des VAH (Verbund für angewandte Hygiene) benutzt wird.

Achtung: Das Desinfektionsmittel muss vor dem Einstechen/Eröffnen verdunstet sein.

Das restliche Untersuchungsmaterial wird bis zu 9 Tage bei $2-10^{\circ} \mathrm{C}$ für eine ggf. erforderliche zweite Kultur gelagert.

\section{Nachweisverfahren und Inokulationsvolumen}

Von jedem Untersuchungsmaterial wird je eine aerobe und anaerobe Kultur ausschließlich mittels automatisierter Kulturverfahren angelegt. Bebrütungstemperatur und Beimpfungsvolumen (4-10 ml pro Kulturmedium) richten sich nach den Vorgaben des Geräteherstellers. Ohne Keimwachstum ist eine Bebrütungsdauer von 7 Tagen einzuhalten.

Bei Vorliegen eines entsprechenden Freigabeprotokolls für die Kulturmedien seitens des Herstellers ist keine zusätzliche Chargenkontrolle beim Anwender erforderlich.

\section{Untersuchungsergebnisse und Bewertung}

Ist im ersten Ansatz der mikrobiologischen Kontrolle kein Keimwachstum nachzuweisen, ist das Ergebnis negativ: Es sind keine weiteren Untersuchungen erforderlich.

Im ersten Ansatz der mikrobiologischen Kontrolle wird Keimwachstum nachgewiesen:

- Es wird eine Subkultur zur Keimdifferenzierung (bis Spezies) angelegt.

- Es wird eine 2. Kultur als Wiederholungsuntersuchung angelegt.

- Bei erneut positivem Ergebnis und Identität des Keims mit dem Ergebnis aus der ersten Kultur (Keimdifferenzierung) ist die Kultur als bestätigt positiv zu bewerten.

- Sind die Erreger nicht identisch oder ist das Ergebnis der 2. Kultur negativ, so ist abzuklären, ob durch methodische Fehler eine Sekundärkontamination verursacht wurde.
Tritt im ersten Ansatz ein positives Signal auf, sind alle verbundenen Blutkomponenten (z. B. weitere TK bei Doppel-/Dreifach-Apherese, verbundene EK bei Pool-TK) von der Transfusion auszuschließen.

Bestätigt positive Befunde bei der mikrobiologischen Kontrolle von Blutkomponenten werden als "außerhalb der Spezifikation" gewertet.

\section{Mikrobiologische Testung von TK als Freigabekriterium}

Werden zur Verlängerung der Haltbarkeit von TK mikrobiologische Schnelltests eingesetzt, so ist trotzdem zusätzlich eine mikrobiologische Stichprobenkontrolle, wie in diesem Votum beschrieben, am Ende der Haltbarkeit durchzuführen, da die Sensitivität der mikrobiologischen Schnellteste begrenzt ist.

Dieses Votum ersetzt das Votum 16 des Arbeitskreises Blut.

Für den Arbeitskreis Blut Prof. Dr. R. Burger, Vorsitzender Dr. R. Offergeld, Geschäftsführerin

Eine englische Fassung dieses Votums ist verfügbar unter

http://www.rki.de $>$ Englisch $>$ Prevention of Infection $>$ National Advisory Committee „Blood“. 